\title{
Reported obstructive sleep apnea does not diminish the cardiometabolic health benefits from a comprehensive lifestyle intervention program
}

\author{
Peter M. Farrehi MD | Robert D. Brook MD ｜ Robert Godley MD | \\ Patrick Walden | Qingmei Jiang | Melvyn Rubenfire MD
}

Division of Cardiovascular

Medicine, University of Michigan Health

System, Ann Arbor, Michigan

\section{Correspondence}

Peter M. Farrehi, Division of Cardiovascular

Medicine, University of Michigan, Ann

Arbor, MI.

Email: pfarrehi@med.umich.edu

\begin{abstract}
Obstructive sleep apnea (OSA) commonly coexists with the metabolic syndrome, a condition for which behavioral changes are often prescribed. Whether OSA diminishes the cardiometabolic health benefits from lifestyle interventions remains unclear. We evaluated 278 consecutive metabolic syndrome participants enrolled in a 12 -week comprehensive lifestyle intervention program. The changes in blood pressure (BP), along with other metabolic health parameters, from baseline to follow-up were compared between those with and without OSA. Mean age was $52.4 \pm 10.9$ years and $37 \%$ were male. At enrollment, mean body mass index (BMI) was $38.2 \pm 7.7 \mathrm{~kg}$ / $\mathrm{m}^{2}$. OSA was reported in 126 of 269 final participants (47\%). At baseline, participants with reported OSA were more likely to be male, older, have a higher BMI, waist and neck size (all $P<0.05)$. At program completion, participants with $(-5.8 \pm 16.1$ / $-3.0 \pm 10.0 \mathrm{~mm} \mathrm{Hg})$ and without OSA $(-4.7 \pm 13.1 /-3.3 \pm 8.2 \mathrm{~mm} \mathrm{Hg})$ had significant reductions in systolic and diastolic BP as well as BMI, fasting glucose, and triglyceride levels. There were no significant differences in the absolute or percentage changes in BP or other metabolic parameters between groups. Our findings support that patients with the metabolic syndrome can derive substantial health benefits, including reductions in $\mathrm{BP}$, by a lifestyle intervention program regardless of the presence of OSA.
\end{abstract}

\section{1 | INTRODUCTION}

Obesity is a rising global epidemic.Excess adiposity poses a tremendous global public health burden in part due to the multitude of associated comorbidities including hypertension, diabetes mellitus (DM) and dyslipidemia. ${ }^{1}$ This constellation of disorders (ie, the metabolic syndrome) substantially increases the risk for cardiovascular morbidity and mortality as well as several other diseases. ${ }^{2-5}$ Unfortunately, the prevalence of the metabolic syndrome, currently impacting $\sim 20 \%$ of the population, is only expected to increase. ${ }^{6}$

Due to shared risk factors (eg, obesity), the prevalence of obstructive sleep apnea (OSA) among patients with the metabolic syndrome often exceeds $50 \%$-with the association becoming stronger as OSA severity increases. ${ }^{7}$ Moreover, the underlying pathophysiology (eg, insulin resistance, sympathetic overactivity, impaired exercise capacity) of OSA and the metabolic syndrome significantly overlaps, worsening cardiovascular function. ${ }^{8,9}$ To help combat the mounting obesity epidemic, a number of lifestyle changes (eg, weight loss) capable of improving both OSA and the metabolic syndrome are commonly recommended. ${ }^{10-13}$ In small studies, the positive impact of lifestyle changes on nerve reflex function was observed regardless of OSA status. ${ }^{14,15}$ However, it is plausible that larger studies may not reveal similar findings. We hypothesized that perturbations in normal metabolic processes, as well as poor sleep, daytime somnolence, and impaired exercise capacity could serve to thwart the benefits of lifestyle interventions. As such, we investigated if OSA influences the cardiometabolic benefits, with a focus on blood pressure (BP) 
reductions, from a 12-week lifestyle intervention program in large group of patients with the metabolic syndrome.

\section{2 | METHODS}

The Metabolic Fitness Program (MetFit) is a prospective, nonrandomized, unblinded, lifestyle intervention program targeting the metabolic syndrome. ${ }^{13}$ Participants are physician referred with components of the metabolic syndrome as defined using American Heart Association (AHA)/NCEP ATP III. Informed consent was obtained on all participants as approved by the University of Michigan Medical School Institutional Review Board. This current study represents a post hoc observational analysis from the data collected as part of the MetFit program. Our objective was to determine if OSA status influences the cardiometabolic health benefits, with a specific focus on the degree of BP reductions.

\section{1 | Patient assessment}

Upon enrollment each participant was evaluated by a nurse practitioner. The baseline assessment includes a medical history, fasting laboratory tests, as well as weight, waist, and neck circumference. BP was measured in the right and left arms in a seated position with arm elevated to the level of the heart. Reported blood pressures are the highest of the two arms. Symptom-limited exercise testing was performed in participants prior to initiating the exercise program if indicated following AHA standards. ${ }^{16}$ Patient assessments, less the symptom-limited stress test, are repeated at 12 weeks.

\section{2 | Program}

The MetFit program is a 12 -week program that utilizes 90 -minute sessions once weekly in a cardiac rehabilitation setting. ${ }^{9}$ Each session includes 45 minutes of group education on cardiovascular risk factor reduction strategies including nutrition, stress management, and exercise. The remaining portion of each weekly session is staff-guided aerobic exercise and group exercise consisting of circuit training, stretching, balance, and strengthening exercises.

\section{3 | Exercise content}

Prior to the first session, each participant had an individualized exercise consultation with an exercise physiologist (ACSM-CEP). The exercise physiologist reviews the fundamentals of an exercise program, barriers to participation, encourages basic goal setting, and crafts an initial exercise prescription. Exercise intensity was individually prescribed based on the symptom-limited stress test (if available) and provided as $60 \%-85 \%$ maximum heart rate achieved (Karvonen formula), ratings of perceived exertion (BORG), and symptoms or orthopedic limitations. Recommendations for exercise include progression from 150 to 300 minutes per week of a combination of moderate and vigorous intensity aerobic activity. ${ }^{17}$

\section{4 | Nutrition content}

The nutrition component of MetFit is based on a Mediterranean food pattern high in fruits, vegetables, heart-healthy fats, and soluble fiber. The education is designed to give participants the information needed to optimize their nutrition choices for improved cardiometabolic parameters, as well as for weight loss of about $5 \%$ at 12 weeks (if appropriate). Each participant receives a baseline caloric intake estimate based on basal metabolic rate estimates created by the registered dietitian and reviewed on the first session. Depending on each participant's level of physical activity the basal metabolic rate is increased by an activity factor to obtain daily intake needs. An in-house, nonvalidated, food frequency questionnaire is obtained by each participant to assess adherence to the Mediterranean food pattern.

\section{5 | Sleep assessment}

During the initial assessment, patients indicated if they had a history of OSA and what if any treatment had been prescribed. Medical record review validated the clinical status of OSA. Patients were excluded if medical record review was inconsistent with data obtained during the history and physical assessment. No polysomnogram data were used in this observational study, and as such, severity and treatment status were not evaluated.

\subsection{Statistical methods}

The Student $t$ tests were used to compare the means in physiologic measures between OSA groups at baseline. Paired $t$ tests and the Student $t$ test were used to examine if there was a statistically significant difference in the changes of physiologic measures within the OSA and non-OSA groups and the difference in the change between each of the two groups. $A P$-value of $<0.05$ was used to determine statistical significance. Data were analyzed using SAS 9.3 (SAS Institute Inc, Cary, NC, USA).

\section{3 | RESULTS}

A total of 278 consecutive metabolic syndrome patients enrolled in a 12-week lifestyle intervention program (Figure 1). Reported OSA ( $n=126,47 \%$ ) was verified by medical record review. Nine participants were excluded after OSA status was unable to be verified through medical record review. Among the participants, 143 did not report a diagnosis of OSA.

Baseline demographics, biometrics, laboratory assays and the differences based on reported OSA status are listed in Table 1. More than $89 \%$ participants were obese $\left(B M I \geq 30 \mathrm{~kg} / \mathrm{m}^{2}\right)$. Program participants attended $8.7 \pm 3.1$ of 12 exercise sessions and $8.5 \pm 3.3$ of 12 education sessions. Reported OSA status did not impact the frequency of participation. Participants who reported a diagnosis of OSA were older, more likely to be male, have a larger BMI, waist and 


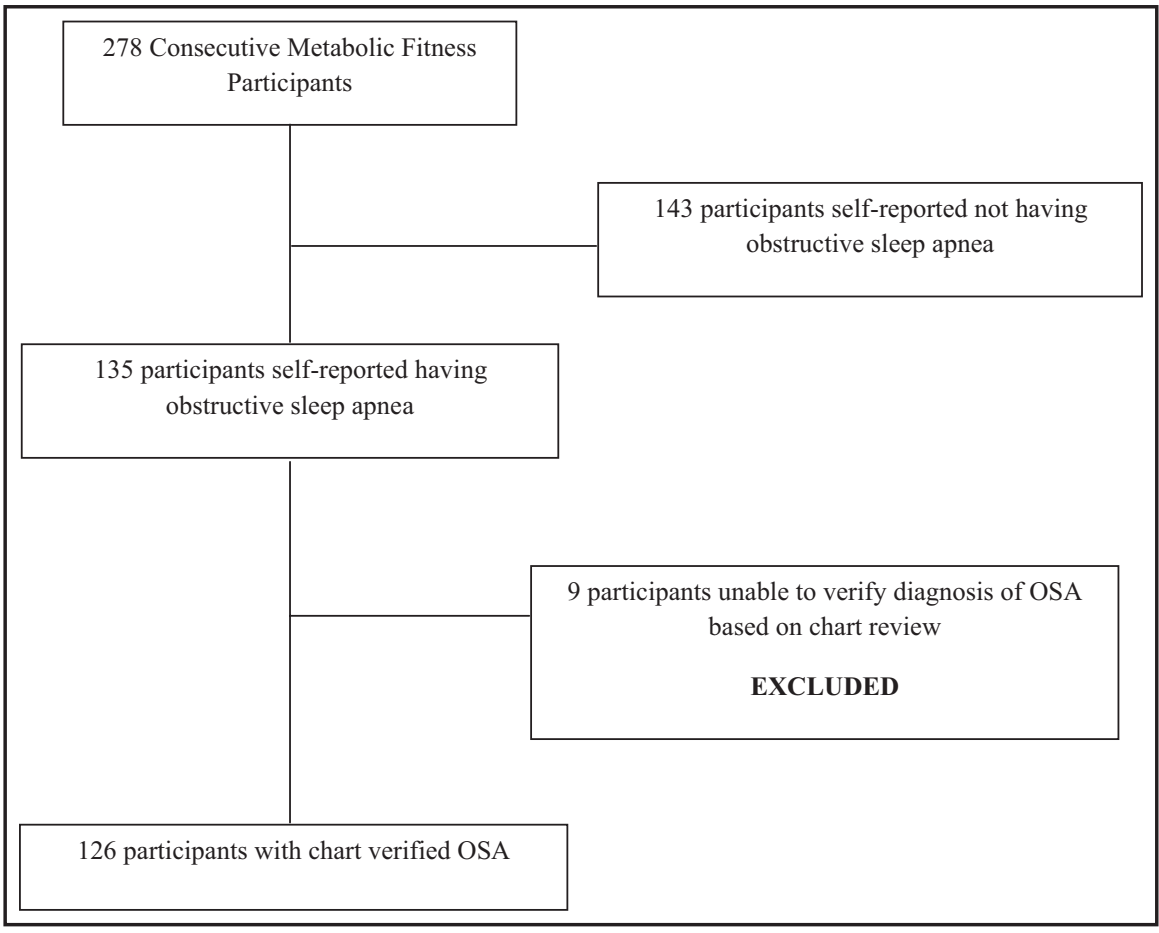

FIGURE 1 Flowchart of the study. Among the nine participants excluded, the diagnosis of OSA could not be verified. Reasons included inability to communicate with referring physicians, lack of electronic medical record interoperability, or refusal to cooperate. OSA, obstructive sleep apnea

neck size. Baseline systolic but not diastolic BP was higher in the OSA group (Table 1).

Many cardiometabolic health parameters improved in both groups following the 12-week lifestyle intervention (Tables 1 and 2). For the entire cohort, mean weight loss at 12 weeks was $2.9 \pm 3.5 \mathrm{~kg}$ and $40.9 \%$ lost more than $5 \%$ of their body weight. For the main study outcome, average systolic and diastolic BP readings fell significantly in the entire cohort $(-5.2 \pm 14.6 /-3.1 \pm 9.0 \mathrm{~mm} \mathrm{Hg})$. Participants with $(-5.8 \pm 16.1 /-3.0 \pm 10.0 \mathrm{~mm} \mathrm{Hg})$ and without OSA $(-4.7 \pm 13.1 /-3.3 \pm 8.2 \mathrm{~mm} \mathrm{Hg})$ had significant reductions in both systolic and diastolic BP levels. Importantly, the magnitude of decreases in BP did not differ between groups (Table 2). Given the differences in baseline values, we also evaluated the percentage change in BP between groups-which also did not significantly differ. In the mixed model analysis, there was no effect modification on the degree of BP reduction by OSA status. At program completion, significantly lower average and percent weight, BMI, and waist size were observed-which were not impacted by OSA status (Table 1). There were numerous additional improvements in cardiometabolic health parameters-none of which were lessened in the OSA group (Table 1).

\section{4 | DISCUSSION}

Our main finding was that while OSA was common among metabolic syndrome patients, it did not negatively impact the cardiometabolic health benefits derived from a comprehensive lifestyle intervention program. Importantly, BP levels were significantly reduced after 12weeks by a similar degree in both patients with and without OSA. Our findings are important because they support that health care providers should recommend to their patients with the metabolic syndrome to undertake these effective lifestyle changes-even in the presence of OSA.

MetFit is an outpatient lifestyle intervention program designed to help patients with the metabolic syndrome adopt sustainable lifestyle modifications and behavior change targeting improved physiologic, metabolic and biochemical profiles, and weight reduction. OSA was highly prevalent among MetFit participants (eg, nearly $50 \%$ ) which is not unexpected given the shared underlying risk factors (ie, obesity). ${ }^{18}$ OSA was associated with a larger BMI, greater waist and neck circumferences, and higher insulin levels compared to those without reported OSA. Despite modestly worse baseline metabolic parameters, patients with reported OSA derived similar overall cardiometabolic health benefits from a comprehensive lifestyle intervention program.

Observed anthropometric and biochemical changes were similar to previous short-term lifestyle interventions. ${ }^{19}$ Improvement in average weight loss was not as significant as programs that included meal replacement. ${ }^{20}$ Systolic and diastolic BP response to weight loss were in expected range of $1 \mathrm{~mm} \mathrm{Hg}$ reduction per kilogram of weight loss. ${ }^{21}$ In our study, gender and OSA status did not impact program participation. Therefore, the observed change in metabolic parameters was not influenced by the degree of participation as measured by session attendance.

Excess weight and OSA are commonly associated. Both randomized and nonrandomized weight loss trials have improved nocturnal events among those with OSA, but rarely prove to eliminate apneas completely. ${ }^{12}$ Here, we evaluated whether the presence of OSA diminishes the effectiveness of a lifestyle intervention program. This is a plausible hypothesis since perturbations in normal metabolic processes, as well as poor sleep, daytime somnolence, and impaired exercise capacity could serve to thwart the benefits. However, we found no such evidence. 
TABLE 1 Baseline health parameters for metabolic fitness participants

\begin{tabular}{|c|c|c|c|c|}
\hline \multirow[b]{2}{*}{ Characteristics } & \multirow[b]{2}{*}{$\begin{array}{l}\text { All participants } \\
(\mathrm{n}=269)\end{array}$} & \multicolumn{3}{|c|}{ Reported OSA status } \\
\hline & & $\begin{array}{l}\text { Negative } \\
(n=143)\end{array}$ & $\begin{array}{l}\text { Positive } \\
(n=126)\end{array}$ & $P$-value \\
\hline \multicolumn{5}{|l|}{ Demographics } \\
\hline Mean age, years & $52.4(10.9)$ & $49.9(11.6)$ & $55.4(9.3)$ & $<0.0001$ \\
\hline Female sex, \% & $168(62.9)$ & $97(67.8)$ & $71(56.4)$ & 0.052 \\
\hline White race,\% & 231 (8686) & $117(82.4)$ & 114 (91.9) & 0.02 \\
\hline Weight, kg & $109.7(24.2)$ & $105.0(21.4)$ & $115.0(26.1)$ & 0.0006 \\
\hline $\mathrm{BMI}, \mathrm{kg} / \mathrm{m}^{2}$ & $38.2(7.7)$ & $36.9(7.6)$ & $39.7(7.6)$ & 0.003 \\
\hline Waist, cm & $116.5(15.7)$ & $112.5(14.1)$ & 120.9 (16.4) & $<0.0001$ \\
\hline Neck size, $\mathrm{cm}$ & $40.5(5.0)$ & $39.3(3.9)$ & $41.9(5.8)$ & $<0.0001$ \\
\hline \multicolumn{5}{|l|}{ Blood pressure } \\
\hline Systolic BP, mm Hg & $121.8(16.4)$ & $119.1(14.9)$ & $124.9(17.6)$ & 0.004 \\
\hline Diastolic BP, mm Hg & $73.6(9.5)$ & $73.6(9.4)$ & $73.5(9.7)$ & 0.9 \\
\hline \multicolumn{5}{|l|}{ Metabolic lab parameters } \\
\hline $\begin{array}{l}\text { Glucose, mg/ } \\
\text { dL-Non-DM }\end{array}$ & $100.5(10.1)$ & $99.8(10.0)$ & $101.3(10.4)$ & 0.3 \\
\hline Glucose, $\mathrm{mg} / \mathrm{dL}-\mathrm{DM}$ & $155.5(54.4)$ & $148.5(45.1)$ & $163.0(62.8)$ & 0.2 \\
\hline $\mathrm{Hgb}$ A1c, \% & $6.3(1.2)$ & $6.3(1.3)$ & $6.4(1.1)$ & 0.8 \\
\hline $\begin{array}{l}\text { Fasting INS, } \mu \mathrm{mol} / \\
\mathrm{mL}-\text { Non-DM }\end{array}$ & $16.5(9.8)$ & $15.5(9.2)$ & $17.7(10.4)$ & 0.1 \\
\hline HOMA-IR-NonDM & $4.1(2.6)$ & $3.9(2.5)$ & $4.5(2.7)$ & 0.1 \\
\hline LDL-C, mg/dL & $105.7(32.9)$ & $107.4(32.6)$ & 103.9 (33.3) & 0.4 \\
\hline $\mathrm{HDL}-\mathrm{C}, \mathrm{mg} / \mathrm{dL}$ & $46.2(12.2)$ & $47.3(11.6)$ & 45.0 (12.7) & 0.1 \\
\hline $\mathrm{TG}, \mathrm{mg} / \mathrm{dL}$ & $182.8(107.6)$ & $181.6(110.3)$ & $184.2(104.9)$ & 0.8 \\
\hline
\end{tabular}

Data are given as number (\%); or mean \pm SD $P$-value represents a comparison of the negative vs positive OSA demographics.

BP, blood pressure; C, cholesterol; HDL, high-density lipoprotein; Hgb, Hemoglobin; HOMA-IR, Homeostatic Model Assessment of Insulin Resistance; INS, insulin; LDL, low-density lipoprotein; OSA, obstructive sleep apnea; TG, triglycerides.

TAB LE 2 Change in blood pressure following lifestyle intervention

\begin{tabular}{|c|c|c|c|c|c|c|c|}
\hline Characteristics & \multicolumn{6}{|c|}{ Reported OSA status } & $\begin{array}{l}\text { Comparison between } \\
\text { OSA groups } \\
P \text {-value }\end{array}$ \\
\hline Systolic BP, mm Hg & $119.1(14.9)$ & $115.6(14.8)$ & 0.0003 & $124.9(17.6)$ & $119.6(15.4)$ & 0.001 & 0.6 \\
\hline
\end{tabular}

Comparison of change in blood pressure at 12-wk follow-up based on OSA presentation.

Data are given as number (\%); or mean \pm SD.

BL, baseline; DBP, diastolic blood pressure; OSA, obstructive sleep apnea; SBP, systolic blood pressure.

${ }^{*} P$-value represents a comparison of the baseline vs 12 -wk treatment results.

${ }^{\pi} P$-value represents a comparison of the change in BP in OSA compared to the change in BP in the non-OSA group.

Hence, the main clinical message from our findings is that OSA should not dissuade health care providers from recommending lifestyle intervention programs to their patients with the metabolic syndrome. Indeed, the presence of OSA should not add to the list of barriers already known to dissuade patients from enrolling in these programs. ${ }^{22}$
Our findings are derived from a post hoc observational analysis of the MetFit program and therefore would benefit from corroboration in a randomized trial. Nevertheless, the sample size of our study was relatively large, and we found no evidence to support even minor differences in health benefits between groups. Even if OSA does in fact 
have a negative impact, we believe our results support that any true differences would be rather modest and not likely of clinical relevance. Motivational bias by self-pay may well have influenced the outcome and may prohibit generalizability to lower socioeconomic groups. The presence and severity of OSA in our study population was not universally assessed with a sleep study, thus the findings are not fully generalizable. Therefore, our study is unable to comment whether mild OSA benefited from lifestyle intervention more or less than severe OSA. However, clinical and biochemical characteristics measured were taken from a large sample size, giving our conclusions strength. An additional limitation was the lack of medical record review to confirm negative OSA status with the participant's initial self-report. It is possible that occult OSA was present in some patients without reported OSA. Finally, one final limitation is treatment status and compliance with prescribed treatment was not assessed, so we are careful not to overreach our conclusions.

\section{5 | CONCLUSIONS}

Lifestyle interventions targeting components of the metabolic syndrome are helpful in reducing BP and other cardiometabolic health parameters even in patients with OSA. Health care providers should strongly recommend that their patients with the metabolic syndrome undergo formal comprehensive lifestyle programs whenever possible, regardless of the presence of OSA.

\section{CONFLICT OF INTEREST}

The authors have no conflicts of interest to report in this work.

\section{REFERENCES}

1. Van Itallie TB. Health implications of overweight and obesity in the United States. Ann Intern Med. 1985;103:983-988.

2. Grundy SM. Metabolic syndrome pandemic. Arterioscler Thromb Vasc Biol. 2008;28:629-636.

3. Grundy SM, Brewer HB Jr, Cleeman JI, et al. Definition of metabolic syndrome: report of the National Heart, Lung, and Blood Institute/ American Heart Association conference on scientific issues related to definition. Circulation. 2004;109:433-438.

4. Gami AS, Witt BJ, Howard DE, et al. Metabolic syndrome and risk of incident cardiovascular events and death: a systematic review and meta-analysis of longitudinal studies. J Am Coll Cardiol. 2007;49:403-414.

5. Malik S, Wong ND, Franklin SS, et al. Impact of the metabolic syndrome on mortality from coronary heart disease, cardiovascular disease, and all causes in United States adults. Circulation. 2004;110:1245-1250.

6. Beltran-Sanchez $\mathrm{H}$, Harhay MO, Harhay MM, McElligott S. Prevalence and trends of metabolic syndrome in the adult U.S. population, 1999-2010. J Am Coll Cardiol. 2013;62:697-703.

7. Drager LF, Lopes HF, Maki-Nunes C, et al. The impact of obstructive sleep apnea on metabolic and inflammatory markers in consecutive patients with metabolic syndrome. PLoS One. 2010;5: e12065
8. Korcarz CE, Stein JH, Peppard PE, Young TB, Barnet JH, Nieto FJ. Combined effects of sleep disordered breathing and metabolic syndrome on endothelial function: the Wisconsin Sleep Cohort study. Sleep. 2014;37:1707-1713.

9. Drager LF, Bortolotto LA, Maki-Nunes C, et al. The incremental role of obstructive sleep apnoea on markers of atherosclerosis in patients with metabolic syndrome. Atherosclerosis. 2010;208:490-495.

10. Lavie CJ, McAuley PA, Church TS, Milani RV, Blair SN. Obesity and cardiovascular diseases: implications regarding fitness, fatness, and severity in the obesity paradox. J Am Coll Cardiol. 2014:63:1345-1354.

11. Knowler WC, Barrett-Connor E, Fowler SE, et al. DM and diabetes prevention program research $\mathrm{G}$. Reduction in the incidence of type 2 diabetes with lifestyle intervention or metformin. N Engl J Med. 2002;346:393-403.

12. Araghi MH, Chen YF, Jagielski A, et al. Effectiveness of lifestyle interventions on obstructive sleep apnea (OSA): systematic review and meta-analysis. Sleep. 2013;36(1553-62):1562A-1562A.

13. Rubenfire $M$, Mollo L, Krishnan $S$, et al. The metabolic fitness program: lifestyle modification for the metabolic syndrome using the resources of cardiac rehabilitation. J Cardiopulm Rehabil Prev. 2011;31:282-289.

14. Maki-Nunes C, Toschi-Dias E, Cepeda FX, et al. Diet and exercise improve chemoreflex sensitivity in patients with metabolic syndrome and obstructive sleep apnea. Obesity (Silver Spring). 2015;23:1582-1590.

15. Toschi-Dias E, Trombetta IC, Silva V, et al. Diet associated with exercise improves baroreflex control of sympathetic nerve activity in metabolic syndrome and sleep apnea patients. Sleep Breath. 2018. https://doi.org/10.1007/s11325-018-1675-x. [Epub ahead of print].

16. Gibbons RJ, Balady GJ, Beasley JW, et al. Guidelines for exercise testing. A report of the American College of Cardiology/American Heart Association Task Force on Practice Guidelines (Committee on Exercise Testing). J Am Coll Cardiol. 1997;30:260-311.

17. Young T, Peppard PE, Taheri S. Excess weight and sleep-disordered breathing. J Appl Physiol. 2005;99:1592-1599.

18. Simpson L, McArdle N, Eastwood PR, et al. Physical inactivity is associated with moderate-severe obstructive sleep apnea. J Clin Sleep Med. 2015;11:1091-1099.

19. Walden P, Jiang Q, Jackson EA, Oral EA, Weintraub MS, Rubenfire $M$. Assessing the incremental benefit of an extended duration lifestyle intervention for the components of the metabolic syndrome. Diabetes Metab Syndr Obes. 2016;9:177-184.

20. Flechtner-Mors M, Ditschuneit HH, Johnson TD, Suchard MA, Adler G. Metabolic and weight loss effects of long-term dietary intervention in obese patients: four-year results. Obes Res. 2000;8: 399-402.

21. Neter JE, Stam BE, Kok FJ, Grobbee DE, Geleijnse JM. Influence of weight reduction on blood pressure: a meta-analysis of randomized controlled trials. Hypertension. 2003;42:878-884.

22. Aurora RN, Collop NA, Jacobowitz O, Thomas SM, Quan SF, Aronsky AJ. Quality measures for the care of adult patients with obstructive sleep apnea. J Clin Sleep Med. 2015;11:357-383.

How to cite this article: Farrehi PM, Brook RD, Godley R, Walden P, Jiang Q, Rubenfire M. Reported obstructive sleep apnea does not diminish the cardiometabolic health benefits from a comprehensive lifestyle intervention program. J Clin Hypertens. 2018;20:1610-1614. https://doi.org/10.1111/ jch.13406 